\title{
AWELS: Adaptive Web-Based Education and Learning Styles
}

\author{
Alexandra Cristea and Natalia Stash \\ Department of Computer Science \\ PO 513, 5600 MB Eindhoven, The Netherlands \\ a.i.cristea@tue.nl;nstash@win.tue.nl
}

\begin{abstract}
Can learning styles (LS) be applied in current webbased systems? What are the main issues when applying LS in web-based systems? Can LS be authored in current web-based systems? What are the main issues when authoring LS in web-based systems? Can LS applications improve the efficiency of learning in web-based systems? Can LS applications be tested in web-based systems? These are important questions that are starting to be asked both in adaptive hypermedia (AH), as well as in web-based learning communities. This paper's aim is to raise the awareness of the timeliness of asking these questions now. Moreover, the paper sketches some preliminary answers, intended for starting a cross-disciplinary debate on these issues.
\end{abstract}

\section{Background and Motivation}

Learners are individuals. Web-based educational research is slowly starting to take that into consideration, moving away from the "one-size-fits-all" approach of educational broadcasting. The move is faster in the research field, where many adaptive educational systems already take into account different learner features like goals/tasks, knowledge, background, hyperspace experience, preferences and interests. In the commercial sphere, giant learning management systems like Blackboard [2] and WebCT [3] don't yet offer personalization. However, SMEs (Small and Medium Sized Enterprises) already are on the lookout for the future market of personalized distance education. Standards, like LOM [5], SCORM [6] or IMS LD specification 0 move already towards introducing snippets of adaptation on a larger scale.

Learning Styles and their effects on learning have been examined most carefully by Coffield [3].

Learning styles (or cognitive styles, CS), as well as the best ways of responding with corresponding instructional strategies, have been intensively studied in the classical educational (classroom) setting. Researchers in this area suggest that matching users' LS with the design of instruction is an important factor with regard to learning outcome. A number of experiments indicate that the user's performance is much better if the teaching methods are matched to the preferred LS. There is much less research of application of LS in the new educational space, created by the Web.

Moreover, authoring applications are scarce, and they do not provide explicit choices and creation of instructional strategies for specific LS. Authoring of Adaptive Web-courses has been long considered as secondary to adaptive course delivery. The authoring task is however not trivial at all. There exist some approaches to help authors to build adaptivehypermedia ( $\mathrm{AH})$-based systems, yet there is a strong need of high-level approaches, formalisms and tools that support and facilitate the description of reusable adaptive web-courses. Only recently have we noticed a shift in interest, as it became clearer that the implementation-oriented approach would forever keep adaptive web-courses away from the 'layman' author. The creator of adaptive courseware cannot be expected to know all facets of this process, but can be reasonably trusted to be an expert in one of them. It is therefore necessary to research and establish the components of an adaptive courseware system from an authoring perspective, catering for the different author personas that are required. This type of research has proven to lead to a modular view on the adaptive hypermedia. It also becomes more and more clear that for Adaptive Web-Environments it is necessary to consider not only the learner's characteristics, but also the pedagogical knowledge to deal with these characteristics.

However, returning to the issue of Learning Styles, just as there are educators and psychologists convinced of their usefulness, there are also adversaries of the appropriateness of applying LS [3, 8]. From this point 
of view, the web learning environment offers an excellent testing bed for the appropriateness of using learning style-based education, due to its potentially extremely large pool of learners, as well as faster response and processing time.

In conclusion, application of LS in adaptive webbased systems is timely, due to the fact that now, both interest in these applications has risen, as well as the initial hardware and software problems have been overcome. Therefore, the main issues that future research has to answer are on authoring, design, implementation, delivery and evaluation of LS in Webbased Education.

\section{Main Questions \& Preliminary Answers}

The above future research issues are reflected in the main questions we set in the abstract. In the following, we are going to give some preliminary answers to these questions.

1) Can learning styles be applied in current webbased systems?

- Yes: There is clear evidence [9] that LS are starting to be implemented in now-a-days web-based systems. It is a slow process, with many hindrances, as presented below.

2) What are the main issues when applying learning styles in web-based systems?

- Timeliness: The technology and access bandwidth of learning systems on the web has progressed sufficiently, so we can implement LS adaptivity (which is more complex).

- Methodology for design, authoring and engineering or LS-based systems, as presented below.

- Usefulness: the usefulness of LS in adaptive web based systems needs to be further investigated.

- Evaluation: see also below.

- Other issues: LS-based collaboration: what are the differences to other types of collaboration?; feedback based on LS: how can it be realized?

3) Can learning styles be authored in current webbased systems?

- Yes: as LS have been integrated into AH - and other web-based systems, they were obviously authored.

- No: there is little research presently on how to smoothen the application process of LS into adaptive educational systems, especially from an authoring point of view [9].

4) What are the main issues when authoring learning styles in web-based systems?

- independent components in the authoring process: these can be used to specialize different authoring personas on authoring only part of the whole material
- difference between general educational adaptive strategies for learning and LS-based strategies, from an implementation point of view

- feedback and automatization to help the author

5) Can learning styles-applications improve the efficiency of learning in web-based systems?

- Yes: studies on the efficiency increase have been made [3]. American schools use LS as default guiding system for years.

6) Can learning styles-applications be tested in webbased systems?

- Yes: again, initial tests exist, but there is no consistent testing procedure that can be applied for different systems. Benchmarks would be beneficial.

\section{Conclusion}

In this paper we outlined the objectives of the current, as well as future research on learning styles in web-based education. We have also given a set of short, somewhat oversimplified answers to the main questions, in order to stimulate future discussions. For the purpose of these discussions we have started AWELS, the "First Workshop on Adaptive Web-based Education and Learning Styles". We hope it will stimulate fruitful discussions between its participants and will contribute to this cross-disciplinary topic.

\section{References}

[1] AWELS, the "First Workshop on Adaptive Web-based Education and Learning Styles", http://www.win.tue.nl/ acristea/AWELS

[2] Blackboard, http://www.blackboard.com/

[3] Coffield, F., Learning Styles and Pedagogy in post-16 learning: A systematic and critical review. Learning \& Skills research centre. http://www.lsda.org.uk/files/pdf/1543.pdf

[4] WebCT, http://www.webct.com/

[5] LOM standard, http://ltsc.ieee.org/wg12/.

[6] SCORM standard, http://www.adlnet.org/index.cfm?fuseaction=scormabt

[7] IMS Global Learning Consortium. "IMS Learning Design Information Model” (IMS-LD), version 1.0 Final Specification, 20 january 2003. http://www.imsproject.org/learningdesign/index.cfm

[8] R. D. Freedman and S. A. Stumpf. Learning style theory: less than meets the eye. Academy of Management Review, 6:297-299, 1980.

[9] Stash, N., Cristea, A., De Bra, P., Authoring of Learning Styles in Adaptive Hypermedia: Problems and Solutions, Proceedings of the WWW 2004 Conference, New York, NY, USA, May 2004, pp. 114-123. 